\title{
Der elektronische Faktor in der Alkanoxidationskatalyse
}

Maik Eichelbaum, ${ }^{*, \dagger, \ddagger}$ Michael Hävecker, ${ }^{\dagger}$, Christian Heine, $^{\dagger}$ Anna Maria Wernbacher, ${ }^{\dagger}$ Frank Rosowski $^{\ddagger}, \S$ Annette Trunschke $^{\dagger}$ and Robert Schlögl $^{\dagger}$

${ }^{\dagger}$ Abteilung Anorganische Chemie, Fritz-Haber-Institut der Max-Planck-Gesellschaft, Faradayweg 4-6, 14195 Berlin (Deutschland), ${ }^{\ddagger}$ BasCat, UniCat BASF JointLab, TU Berlin, Marchstraße 6, 10587 Berlin (Deutschland), "Helmholtz-Znetrum Berlin / BESSY II, Energiekatalyse, Albert-Einstein-Straße 15, 12489 Berlin (Deutschland), and ${ }^{\S}$ Process Research and Chemical Engineering, Heterogeneous Catalysis, BASF SE, Carl-Bosch-Straße 38, 67056 Ludwigshafen (Deutschland)

E-mail: me@fhi-berlin.mpg.de

Phone: +49 (0)30 84134566. Fax: +49 (0)30 84134405

\footnotetext{
* To whom correspondence should be addressed

${ }^{\dagger}$ Fritz-Haber-Institut

${ }^{\ddagger}$ BasCat, UniCat BASF JointLab

『Helmholtz-Zentrum Berlin / BESSY II

$\S$ BASF SE
} 
zu Maleinsäureanhydrid über dem hochselektiven Katalysator Vanadylpyrophosphat und der mäßig selektiven $\mathrm{MoVTeNbO}_{x}$ M1-Phase anhand der Gasphasenabhängigkeit der Austrittsarbeit, Elektronenaffinität und der Oberflächenpotentialbarriere gezeigt werden, dass sich diese Katalysatoren wie halbleitende Gassensoren mit einem dynamischen Ladungstransfer zwischen Volumen und Oberfläche verhalten. Im Gegensatz dazu hat die Gasphase nur einen geringen Einfluss auf die halbleitenden Eigenschaften des Verbrennungskatalysators $\mathrm{V}_{2} \mathrm{O}_{5}$ und es wurde in diesem Fall keine dynamische Oberflächenpotentialbarriere beobachtet. Das Oberflächenpotential wird demnach als Deskriptor für selektive Katalysatoren vorgeschlagen.

Seit der Mitte des letzten Jahrhunderts wurden Konzepte aus der Halbleiterphysik vorgeschlagen, um die Arbeitsweise selektiver Alkan- und Alkenoxidationskatalysatoren zu beschreiben. ${ }^{1-7}$ Die Vision dieses Ansatzes bestand und besteht darin, die katalytische Aktivität und Selektivität potentieller Katalysatormaterialien in verschiedenen Reaktionen auf Basis ihrer elektronischen Struktur (dem sogenannten "elektronischen Faktor") vorhersagen zu können. Zieht man halbleiterphysikalische Konzepte zur Beschreibung der Adsorbat-KatalysatorWechselwirkung heran, so ergibt sich die Triebkraft für einen Ladungstransfer über die Volumen-Oberfläche-Adsorbat-Grenzfläche aus der Differenz zwischen dem Fermi-Potential des halbleitenden Katalysators und dem Redoxpotential des Adsorbats. ${ }^{5,8}$ Dieser Ladungstransfer verursacht wiederum einen Potentialgradienten und demzufolge ein elektrisches Feld zwischen der Oberfläche und dem Festkörpervolumen und kann leitende Kanäle mit Stromgleichrichtenden Eigenschaften wie an einer p/n-Halbleiter-Kontaktstelle bewirken. ${ }^{9}$ Die Höhe der dadurch gebildeten Oberflächenpotentialbarriere, welche Ladungsträger auf ihrem Weg vom Volumen zur Oberfläche überwinden müssen, kann einen großen Einfluss auf die Kinetik der Oxidationsreaktion auf der Oberfläche und der Aktivierung von Sauerstoff haben. ${ }^{5}$ Das Oberflächenpotential unter Reaktionsbedingungen könnte demzufolge ein geeigneter Deskriptor für die katalytische Leistungsfähigkeit von Oxidationskatalysatoren sein. Bisher steht jedoch noch der eindeutige experimentelle Nachweis aus, ob Oxidation- 
skatalysatoren unter Reaktionsbedingungen überhaupt als halbleitende Gassensoren unter Ausbildung einer gasphasenabhängigen Oberflächenpotentialbarriere beschrieben werden können.

Von generellem Interesse ist das Verständnis der Arbeitsweise des VanadylpyrophosphatKatalysators (VPP). VPP wird kommerziell als Katalysator für die Oxidation von n-Butan zu Maleinsäureanhydrid eingesetzt ${ }^{10-14}$ und gilt als Referenzsystem in der selektiven Alkanoxidationskatalyse ${ }^{15-21}$ welche wiederum im Kontext des bevorstehenden Rohstoffwandels zu einer der bedeutendsten heterogen katalysierten Reaktionsklassen zählt. ${ }^{22}$ Aufgrund der erhöhten elektronischen Leitfähigkeit an Luft und abnehmenden Leitfähigkeit in n-Butanhaltigen Gasmischungen wurde VPP als p-Halbleiter mit Elektronenlöchern als Majoritätsladungsträgern identifiziert. ${ }^{10-14,23-26}$ Allerdings ist ein reversibles Leitfähigkeitsverhalten allein offensichtlich kein ausreichender Deskriptor für selektive Katalysatoren, da auch die Leitfähigkeit von Vanadium(V)-oxid reversibel auf die Gasphase und Reaktionsbedingungen reagiert, jedoch ausschließlich die vollständige Verbrennung von n-Butan zu $\mathrm{CO}_{2}$ (und CO) katalysiert.

In unserem Beitrag berichten wir über die erfolgreiche Anwendung der in-situ RöntgenPhotoelektronenspektroskopie (near-ambient pressure XPS, NAP-XPS), um den Einfluss der reaktiven Gasphase auf die Oberflächenpotentialbarriere in VPP unter n-Butanoxidationsbedingungen mit nachgewiesener Produktion von Maleinsäureanhydrid zu untersuchen. Die Ergebnisse zeigen, dass der Transfer von Ladungsträgern zwischen dem Festkörpervolumen und der Oberfläche mit Hilfe der bisher nur theoretisch von Boudart, ${ }^{1}$ Schwab, ${ }^{2}$ Volkenshtein, ${ }^{3}$ und Morrison $^{4,5}$ beschriebenen Halbleiterkonzepte erklärt werden kann. Wir vergleichen dieses Ergebnis mit dem elektronischen Verhalten des unselektiven Oxidationskatalysators $\mathrm{V}_{2} \mathrm{O}_{5}$ und dem mäßig selektiven Katalysator $\mathrm{MoVTeNbO}_{x}$ (orthorhombische M1-Phase), um ein generell gültiges Konzept zur Erklärung katalytischer Selektivität zu finden.

Der polykristalline Katalysator VPP wurde mit NAP-XPS bei $25 \mathrm{~Pa}$ und $400^{\circ} \mathrm{C}$ in 1:10 Mischungen von n-Butan/Sauerstoff $\left(\mathrm{C}_{4} \mathrm{H}_{10} / \mathrm{O}_{2}\right)$, Helium/Sauerstoff $\left(\mathrm{O}_{2}\right)$ und n-Butan/Helium 
$\left(\mathrm{C}_{4} \mathrm{H}_{10}\right)$ entsprechend dem in den Hintergrundinformationen beschriebenen Protokoll untersucht. Der verwendete VPP-Katalysator produziert in einem Festbett-Durchfluss-Reaktor bei 1 bar Maleinsäurenanhydrid mit einer Selektivität zwischen 70 und $80 \% .{ }^{24,25}$ In der Halbleiterphysik kann eine gasphasenabhängige Oberflächenpotentialbarriere (Bandbiegung) und demnach ein Austausch von Ladungsträgern zwischen der Volumen- und Oberflächenphase nachgewiesen werden, wenn die Austrittsarbeit, die Valenzbandenergie und die Bindungsenergien sämtlicher Rumpfelektronen konsistent um denselben absoluten Betrag bei Adsorption verschiedener Gase verschoben werden (siehe auch Abbildung S2 in den Hintergrundinformationen). ${ }^{8}$ Daher wurde die Abhängigkeit der Valenzbandkante, des V3d-Valenzzustands, der Austrittsarbeit (durch Messung der Sekundärelektronenabrisskante) und der O1s-, V2pund P2p-Rumpfelektronenniveaus von VPP in den verschiedenen Gasmischungen gemessen. Abbildung 1 zeigt Photoelektronenspektren bei hohen (Sekundärelektronenabrisskante) und niedrigen Energien (Valenzband) in den drei verschiedenen Gasatmosphären. Das Maximum bei ca. 2-2.5 eV unterhalb des Fermi-Niveaus $(0 \mathrm{eV})$ und neben dem Valenzband wird einem besetzten Vanadium 3d-Zustand zugeschrieben. Es wird beobachtet, dass die Sekunärelektronenabrisskante, die Valenzbandkante und der V3d-Zustand zu höheren Bindungsenergien unter reduzierenden $\mathrm{C}_{4} \mathrm{H}_{10}$ Bedingungen verschoben werden, während nur geringe Unterschiede in den Spektren zwischen der $\mathrm{C}_{4} \mathrm{H}_{10} / \mathrm{O}_{2}$ und $\mathrm{O}_{2}$ Atmosphäre wahrgenommen werden können. Weiterhin fällt auf, dass die Intensität des dem V3d-Zustand zugeschriebenen Teils des Spektrums in $\mathrm{C}_{4} \mathrm{H}_{10}$ ansteigt. Dies zeigt eine höhere Besetzung dieses Zustands mit Elektronen und demnach eine Reduktion des Katalysators an.

In Abbildung 2 sind die beobachteten Änderungen der Austrittsarbeit, des V3d-Zustands, des Vanadiumoxidationszustands (erhalten aus dem V2p $3 / 2^{-}$Spektrum) und der Elektronenaffinität zusammengefasst. Die Bindungsenergieverschiebungen der Rumpfelektroneniveaus $\mathrm{V}_{2} \mathrm{p}_{3 / 2}$, O1s und P2p sind in Abbildung S1 in den Hintergrundinformationen dargestellt. Es ist ersichtlich, dass der V3d-Zustand reversibel um bis zu $540 \mathrm{meV}$ und die Rumpfelektronenniveaus um ca. $500 \mathrm{meV}$ zwischen der oxidierenden $\mathrm{O}_{2}$ und reduzierenden $\mathrm{C}_{4} \mathrm{H}_{10}$ Gasphase 
verschoben werden. Dieses konsistente Verhalten ist ein starkes Indiz für einen Ladungstransfer zwischen Volumen und Oberfläche und der damit einhergehenden Ausbildung einer Raumladungszone sowie einer gasabhängigen Oberflächenpotentialbarriere (d.h. dem Auftreten einer Bandbiegung). Das Protonentransfer-Massenspektrometrie-(PTR-MS)Signal bei m/z=99 (Masse von protoniertem Maleinsäureanhydrid), aufgenommen während des NAP-XPS-Experiments (Abbildung 2a), beweist, dass unter $\mathrm{C}_{4} \mathrm{H}_{10} / \mathrm{O}_{2}$ Reaktionsbedingungen Maleinsäureanhydrid produziert wird und demzufolge der Katalysator tatsächlich unter katalytischen Arbeitsbedingungen untersucht wurde.

Die Austrittsarbeit $\Phi$ wird aus der Differenz der Anregungsenergie und der Energie an der Hälfte des Maximalwerts der Sekundärelektronenabrisskante berechnet. Die ermittelten Austrittsarbeiten liegen für VPP zwischen $6.94 \mathrm{eV}$ in $\mathrm{O}_{2}$ and $6.70 \mathrm{eV}$ in $\mathrm{C}_{4} \mathrm{H}_{10}$ (Abbildung 2b). Diese Werte liegen in der Nähe der Austrittsarbeit des binären Oxids $\mathrm{V}_{2} \mathrm{O}_{5}(\Phi=7.0 \mathrm{eV}) .{ }^{27}$ Wie bereits zuvor erwähnt, kann die Ausbildung einer Oberflächenpotentialbarriere aufgrund der Angleichung des Fermi-Potentials an das Potential eines Adsorbat-induzierten Oberflächenzustands nachgewiesen werden, wenn die Austrittsarbeit und sämtliche Valenz- und Rumpfelektronenniveaus um denselben Energiebetrag während der Adsorption verschiedener Gase verschoben werden. Allerdings ist die gemessene maximale Änderung von $\Phi$ mit 240 meV signifikant kleiner als die bei den Valenz- und Rumpfelektronenniveaus gemessenen Verschiebungen. Adsorbate können jedoch auf Oberflächen nicht nur elektronische Oberflächenzustände induzieren, die eine Bandbiegung verursachen, sondern gleichfalls die dipolare Oberflächenstruktur beeinflussen. ${ }^{8}$ Dieser Effekt würde sich in einer veränderten Oberflächenelektronenaffinität bemerkbar machen (Abbildung S2 in den Hintergrundinformationen). Weil nun die Energieverschiebungen in den verschiedenen Gasmischungen bei der Austrittsarbeit und den Valenz-/Rumpfelektronenbindungsenergien unterschiedlich sind, haben die Adsorbate auf VPP sowohl Obeflächendipole als auch Oberflächenzustände modifiziert. ${ }^{8}$ Aus dieser Differenz in den Verschiebungen kann die Adsorbat-induzierte Veränderung der Elektronenaffinität $\Delta \chi$ direkt berechnet werden (Abbildung 2e). 
Ein weiteres Ergebnis ist die gasabhängige Veränderung des Vanadiumoxidationszustands, welcher mit fast 4.4 am höchsten in $\mathrm{O}_{2}$ und mit 4.0 am niedrigsten in $\mathrm{C}_{4} \mathrm{H}_{10}$ ist (Abbildung 2d). Weil die Rumpfelektronenspektren bei einer kinetischen Energie aufgenommen wurden, welche einer mittleren freien Elektronenweglänge von $0.7 \mathrm{~nm}$ entspricht und somit weitgehend die erste(n) Oberflächenlage(n) umfasst, ist die Oberfläche von VPP offensichtlich unter $\mathrm{O}_{2}$ und $\mathrm{C}_{4} \mathrm{H}_{10} / \mathrm{O}_{2}$ Bedingungen oxidiert im Vergleich zum Oxidationszustand VPP im Volumen, wo der Vanadiumoxidationszustand 4.0 beträgt.

Die erhaltenen Resultate für das Verhalten der elektronischen Struktur von VPP in den verschiedenen Gasphasen sind schematisch in einem vereinfachten Banddiagramm dargestellt (Abbildung 3). Es ist zu beachten, dass die elektronische Struktur nicht notwendigerweise durch delokalisierte Bänder beschrieben werden muss. Im Gültigkeitsbereich der FermiDirac-Elektronenstatistik können zur Beschreibung der Struktur auch lokalisierte Zustände/Molekülorbitale herangezogen werden und die Leitfähigkeit durch aktivierte Hüpfprozesse von Ladungsträgern zwischen diesen lokalen Zuständen erklärt werden. Auch in diesem Fall könnte sich eine elektrochemische Doppelschicht und ein elektrisches Feld zwischen Oberfläche und Volumen ausbilden, welches sich ebenfalls in einer entsprechenden Verschiebung der Bindungsenergien der Valenz- und Rumpfelektronenniveaus bemerkbar machen würde.

Wie zuvor erwähnt, werden die Bandbiegung und die Oberflächenpotentialbarriere durch die Angleichung des Fermi-Potentials an das Oberflächenzustandspotential verursacht, welches wiederum vom chemischen Potential der Gasphase abhängt. Die Oberflächenzustände auf VPP könnten mit einem $\mathrm{V}^{4+} / \mathrm{V}^{5+}$ Redoxpaar auf der Oberfläche beschrieben werden. In diesem Fall wäre die Fermi-Energie $\left(E_{F}\right)$ durch folgende Gleichung gegeben: ${ }^{5}$

$$
E_{F}(\text { mit Oberflächenzuständen })=E_{t}+k T \ln \frac{\left[\mathrm{V}^{4+}\right]}{\left[\mathrm{V}^{5+}\right]}
$$

$E_{t}$ könnte näherungsweise durch die Redoxenergie des $\mathrm{V}^{4+} / \mathrm{V}^{5+}$ Redoxpaares beschrieben 
werden. Die vorgeschlagene Beziehung zwischen der Oberflächenbarriere (Bandbiegung) und dem Vanadiumoxidationszustand ist aufgrund der simultan zur Bindungsenergieverschiebung beobachteten Modulation des durchschnittlichen Vanadiumoxidationszustands (Abbildung 2d) und der Intensität des V3d-Valenzzustands (Abbildung 1) plausibel. Die Höhe der Oberflächenpotentialbarriere $q V_{B}$ ist durch die Differenz zwischen den Fermi-Energien des Katalysators ohne Oberflachenzustände und mit Oberflächenzuständen gegeben, wobei letztere wiederum durch die Gasphase, wie in Gleichung 1 beschrieben, modifiziert werden:

$$
q V_{B}=E_{F}(\text { ohne Oberflächenzustände, Flachband })-E_{F}(\text { mit Oberflächenzuständen })
$$

Aktuelle oberflächenempfindliche Experimente an VPP unter Reaktionsbedingungen haben gezeigt, dass der Katalysator durch eine zweidimensionale Vanadium(IV,V)-oxidschicht terminiert ist, die deutlich von der Volumenkristallstruktur abweicht. ${ }^{24,25,28-30}$ Die vorgeschlagenen $\mathrm{V}^{4+} / \mathrm{V}^{5+}$ Oberflächenzustände können demnach als Teil dieser Terminierung betrachtet werden, bzw. in der Nomenklatur der Halbleiterphysik, ${ }^{5}$ als extrinsische Oberflächenzustände einer Oberflächenterminierung mit gebrochener Translationssymmetrie bezeichnet werden. Eine solche Terminierung von Katalysatoren wurde bereits allgemein von Boudart in seinem Konzept eines "defect one-phase surface system" vorgeschlagen. ${ }^{1}$

Aus den Gleichungen 1 und 2 ergibt sich, dass ein hohes $\mathrm{V}^{5+} / \mathrm{V}^{4+}$ Verhältnis die Oberflächenpotentialbarriere $q V_{B}$ derart erhöhen kann, dass der Transport von Elektronen vom Volumen zur Oberfläche stark (kinetisch) gehemmt wird. Unter solchen Bedingungen ist demzufolge auch die Aktivierung (Reduktion) von Sauerstoff aus der Gasphase stark beeinträchtigt. Als Konsequenz folgt, dass das Oberflächenpotential die Konzentration an aktiviertem Sauerstoff auf der Oberfläche kontrollieren kann. Da eine Anreicherung aktiven Sauerstoffs auf der Oberfläche zu einer vermehrten Totaloxidation des gewünschten Oxygenats zu $\mathrm{CO}_{x}$ führt, könnte die Oberflächenpotentialbarriere auch die katalytische Selek- 
tivität beeinflussen.

Um diese vorgeschlagenen Beziehungen zwischen dem Oberflächenpotential und der Selektivität zu überprüfen, wurde der unselektive (d.h. ohne Selektivität zu Maleinsäureanhydrid, sondern nur zu $\mathrm{CO}$ und $\mathrm{CO}_{2}$ ) Katalysator Vanadium(V)-oxid ebenfalls mit NAP-XPS untersucht. Unter stark oxidierenden Bedingungen in $\mathrm{O}_{2}$ wurde eine maximale Austrittsarbeit von $7.00 \mathrm{eV}$ und ein Vanadiumoxidationszustand von 4.9 gemessen (Abbildung 4a). Eine Reduzierung des Vanadiumoxidationszustands zu 4.8 wird in $\mathrm{C}_{4} \mathrm{H}_{10}$ beobachtet $(\mathrm{Ab}-$ bildung 4a). Die Austrittsarbeit hat sich unter diesen Bedingungen allerdings nur leicht auf $6.96 \mathrm{eV}$ verringert. Auch die Valenzbandkante hat sich nur gering von $2.18 \mathrm{eV}\left(\mathrm{O}_{2}\right)$ nach $2.22 \mathrm{eV}\left(\mathrm{C}_{4} \mathrm{H}_{10}\right)$ verschoben. Obwohl all diese Veränderungen reversibel und somit real sind, ist auffällig, dass die Differenzwerte zwischen den Bedingungen deutlich kleiner als beim VPP ausfallen. Demzufolge hat die Gasphase nur einen geringen Einfluss auf die Oberflächenpotentialbarriere. Dies kann zunächst durch die deutlich höhere Leitfähigkeit von $\mathrm{V}_{2} \mathrm{O}_{5}$ erklärt werden. ${ }^{31}$ Aufgrund der vergleichsweise hohen Ladungsträgerdichte ist die relative Verarmung an Ladungsträgern im Volumen aufgrund des Ladungstransfers zwischen Katalysator und Gasphase vernachlässigbar klein, so dass sich keine Oberflächenpotentialbarriere und ausgedehnte Raumladungszone ausbildet oder modifiziert wird. Dies hat zur Folge, dass die Sauerstoffaktivierung nicht durch das Oberflächenpotential limitiert ist. Dadurch könnte die beobachtete Totaloxidation von n-Butan zu $\mathrm{CO}_{x}$ auf $\mathrm{V}_{2} \mathrm{O}_{5}$ erklärt werden.

Des Weiteren wurde der mäßig selektive n-Butanoxidationskatalysator $\mathrm{MoVTeNbO}_{x}$ (orthorhombische M1-Phase) untersucht, der eine Maleinsäureanhydrid-Selektivität von mehr als $40 \%$ aufweist. ${ }^{32}$ In diesem Fall wurden als Bedingungen 1:2 Mischungen aus Ethan $/ \mathrm{O}_{2}$ und n-Butan $/ \mathrm{O}_{2}$ gewählt, um eine stärker oxidierende bzw. reduzierende Atmosphäre zu gewährleisten. In $\mathrm{O}_{2}$ oder n-Butan/Helium-Mischungen ist dieser Katalysator unter reduziertem Druck nicht stabil. Zwischen den angewandten Bedingungen veränderte sich der Vanadiumoxidationszustand reversibel zwischen 4.6 und 4.5 (die Oxidationszustände der übrigen Metalle blieben gleich ${ }^{32}$ ), die Austrittsarbeit verschob sich um $200 \mathrm{meV}$ und 
die Valenzbandkante um $70 \mathrm{meV}$ (Abbildung 4b). Dieses Resultat weist demnach auf eine gasabhängige Oberflächenpotentialbarriere sowie eine Änderung der Oberflächenelektronenaffinität um ca. $130 \mathrm{meV}$ hin. Da diese Veränderungen signifikant größer sind als bei $\mathrm{V}_{2} \mathrm{O}_{5}$, und das obwohl bei $\mathrm{MoVTeNbO}_{x}$ die Oxidations-/Reduktionsbedingungen deutlich milder geändert wurden, sind diese Ergebnisse in Übereinstimmung mit der moderaten Selektivität dieses Katalysators und unterstützen das Konzept des Oberflächenpotential-abhängigen Volumen-Oberflächenladungstransfers und dessen Einflusses auf die katalytische Selektivität. Die deutliche Umstrukturierung der Oberfläche unter Reaktionsbedingungen, die bei beiden selektiven Katalysatoren durch die Änderung der Elektronenaffinität angezeigt wird, ist in Übereinstimmung mit der Theorie, dass sich die aktive Oberfläche erst unter Reaktionsbedingungen ausbildet und wird allgemein bei selektiven Oxidationskatalysatoren beobachtet. ${ }^{33}$

Zusammenfassend lässt sich sagen, dass mit NAP-XPS bewiesen wurde, dass die selektiven Alkanoxidationskatalysatoren VPP and $\mathrm{MoVTeNbO}_{x}$ M1-phase wie halbleitende Gassensoren unter Reaktionsbedingungen mit einer gasphasenabhängigen Oberflächenpotentialbarriere reagieren. Diese Ergebnisse können demnach als experimenteller Beweis für die frühen Halbleiterkonzepte zur Beschreibung von Katalysatoren von Boudart, ${ }^{1}$ Schwab, ${ }^{2}$ und Volkenshtein ${ }^{3}$ gelten. Die Ausbildung einer dynamischen Oberflächenpotentialbarriere könnte zudem eine rationale Erklärung für die katalytische Selektivität in Oxidationsreaktionen liefern. Demzufolge könnte die Oberflächenbarriere den Transfer von Ladungsträgern zwischen Volumen und Oberfläche des Katalysators und demzufolge auch die Aktivierung von Sauerstoff auf der Oberfläche kontrollieren. Diese Interpretation wird durch entsprechende Experimente am unselektiven Katalysator $\mathrm{V}_{2} \mathrm{O}_{5}$ unterstützt, wo der Effekt der Gasphase auf die Oberflächenpotentialbarriere sehr gering war.

\section{Danksagung}

Diese Arbeit wurde in Zusammenarbeit mit dem BasCat JointLab der BASF SE, der TU Berlin, des FHI und des Exzellenzclusters UniCat durchgeführt. Den Mitarbeitern des HZB 
wird gedankt für Ihre kontinuierliche Unterstützung der Elektronenspektroskopie-Aktivitäten des FHI am BESSY II.

\section{References}

(1) Boudart, M. J. Am. Chem. Soc. 1952, 74, 1531-1535.

(2) Schwab, G.-M. Angew. Chem. 1961, 73, 399-401.

(3) Volkenshtein, F. F. The Electronic Theory of Catalysis on Semiconductors; Pergamon Press, 1963.

(4) Morrison, S. R. J. Catal. 1974, 34, 462-478.

(5) Morrison, S. R. The Chemical Physics of Surfaces; Plenum Press, 1977.

(6) Haber, J.; Witko, M. J. Catal. 2003, 216, 416-424.

(7) Herrmann, J. M. Catal. Today 2006, 112, 73-77.

(8) Mönch, W. J. Vac. Sci. Technol. B 1989, 7, 1216-1225.

(9) Lüth, H. Space Charge Layer; Springer Verlag, 2001; Chapter Electronic structure of Surfaces, pp $329-380$.

(10) Rouvet, F.; Herrmann, J. M.; Volta, J. C. J. Chem. Soc. Faraday Trans. 1994, 90, $1441-1448$.

(11) Herrmann, J. M.; Vernoux, P.; Bere, K. E.; Abon, M. J. Catal. 1997, 167, 106-117.

(12) Ait-Lachgar, K.; Tuel, A.; Brun, M.; Herrmann, J. M.; Krafft, J. M.; Martin, J. R.; Volta, J. C.; Abon, M. J. Catal. 1998, 177, 224-230.

(13) Rihko-Struckmann, L. K.; Ye, Y.; Chalakov, L.; Suchorski, Y.; Weiss, H.; Sundmacher, K. Catal. Lett. 2006, 109, 89-96. 
(14) Sartoni, L.; Delimitis, A.; Bartley, J. K.; Burrows, A.; Roussel, H.; Herrmann, J. M.; Volta, J. C.; Kiely, C. J.; Hutchings, G. J. J. Mater. Chem. 2006, 16, 4348-4360.

(15) Centi, G.; Trifiro, F.; Ebner, J. R.; Franchetti, V. M. Chem. Rev. 1988, 88, 55-80.

(16) Centi, G. Catal. Today 1993, 16, 5-26.

(17) Volta, J. C. Comptes Rendus De L Academie Des Sciences Serie Ii Fascicule C-chimie 2000, 3, 717-723.

(18) Grasselli, R. K. Top. Catal. 2001, 15, 93-101.

(19) Ballarini, N.; Cavani, F.; Cortelli, C.; Ligi, S.; Pierelli, F.; Trifiro, F.; Fumagalli, C.; Mazzoni, G.; Monti, T. Top. Catal. 2006, 38, 147-156.

(20) Dummer, N. F.; Bartley, J. K.; Hutchings, G. J. Adv. Catal. 2011, 54, 189-247.

(21) Védrine, J. C.; Hutchings, G. J.; Kiely, C. J. Catal. Today 2013, 217, 57-64.

(22) Cavani, F. Catal. Today 2010, 157, 8-15.

(23) Eichelbaum, M.; Stößer, R.; Karpov, A.; Dobner, C.-K.; Rosowski, F.; Trunschke, A.; Schlögl, R. Phys. Chem. Chem. Phys. 2012, 14, 1302-1312.

(24) Eichelbaum, M.; Hävecker, M.; Heine, C.; Karpov, A.; Dobner, C. K.; Rosowski, F.; Trunschke, A.; Schlögl, R. Angew. Chem. Int. Ed. 2012, 51, 6246-6250.

(25) Eichelbaum, M.; Hävecker, M.; Heine, C.; Karpov, A.; Dobner, C. K.; Rosowski, F.; Trunschke, A.; Schlögl, R. Angew. Chem. 2012, 124, 6350-6354.

(26) Eichelbaum, M.; Glaum, R.; Hävecker, M.; Wittich, K.; Heine, C.; Schwarz, H.; Dobner, C. K.; Welker-Nieuwoudt, C.; Trunschke, A.; Schlögl, R. ChemCatChem 2013, 5, $2318-2329$.

(27) Meyer, J.; Zilberberg, K.; Riedl, T.; Kahn, A. J. Appl. Phys. 2011, 110, 033710. 
(28) Kleimenov, E.; Bluhm, H.; Hävecker, M.; Knop-Gericke, A.; Pestryakov, A.; Teschner, D.; Lopez-Sanchez, J. A.; Bartley, J. K.; Hutchings, G. J.; Schlögl, R. Surf. Sci. 2005, 575, 181-188.

(29) Hävecker, M.; Mayer, R. W.; Knop-Gericke, A.; Bluhm, H.; Kleimenov, E.; Liskowski, A.; Su, D.; Follath, R.; Requejo, F. G.; Ogletree, D. F.; Salmeron, M.; Lopez-Sanchez, J. A.; Bartley, J. K.; Hutchings, G. J.; Schlögl, R. J. Phys. Chem. B 2003, 10\%, 4587-4596.

(30) Bluhm, H.; Hävecker, M.; Kleimenov, E.; Knop-Gericke, A.; Liskowski, A.; Schlögl, R.; Su, D. S. Top. Catal. 2003, 23, 99-107.

(31) Heine, C.; Girgsdies, F.; Trunschke, A.; Schlögl, R.; Eichelbaum, M. Appl. Phys. A: Mater. Sci. Process. 2013, 112, 289-296.

(32) Heine, C.; Hävecker, M.; Sanchez-Sanchez, M.; Trunschke, A.; Schlögl, R.; Eichelbaum, M. . J. Phys. Chem. C 2013, 117, 26988-26997.

(33) Schlögl, R. Top. Catal. 2011, 54, 627-638. 


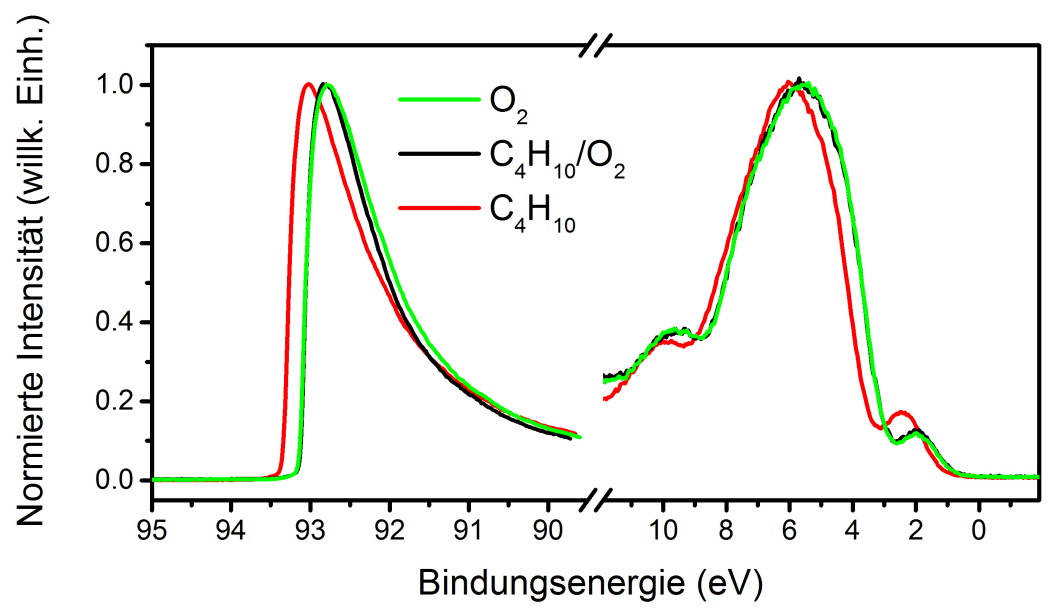

Figure 1: In-situ Röntgen-Photoelektronenspektren im Hoch- (Sekundärelektronenabrisskante; links) und Niedrigenergiebereich (Valenzband mit V3d-Zustand bei ca. $2 \mathrm{eV}$; rechts) von VPP bei $400^{\circ} \mathrm{C}$ unter katalytischen Reaktions-, $\left(\mathrm{C}_{4} \mathrm{H}_{10} / \mathrm{O}_{2}\right)$, oxidierenden $\left(\mathrm{O}_{2}\right)$ und reduzierenden Bedingungen $\left(\mathrm{C}_{4} \mathrm{H}_{10}\right)$. Die Spektren wurden mit einer Anregungsenergie von $100 \mathrm{eV}$ aufgenommen. 


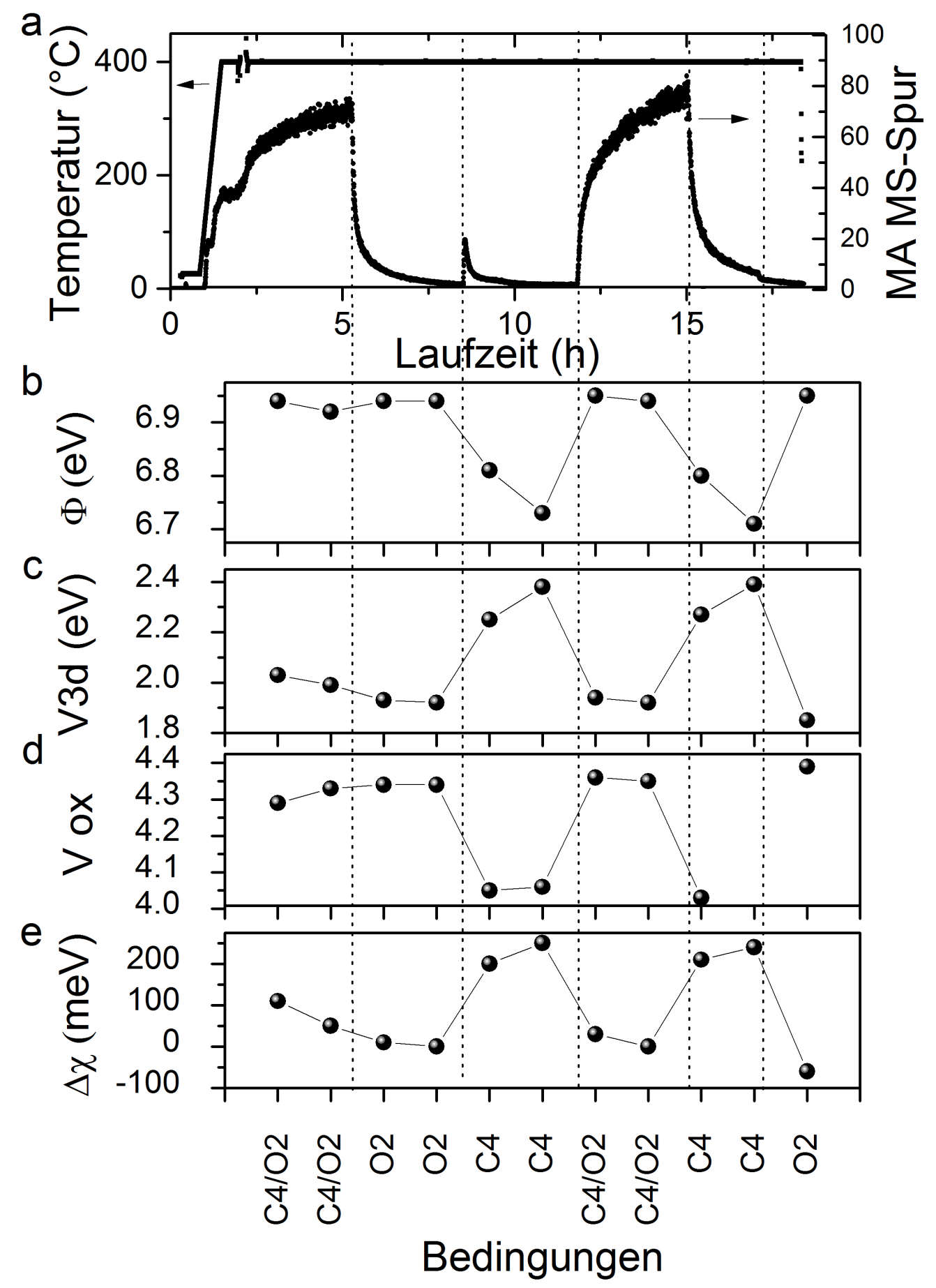

Figure 2: a) PTR-MS-Spur von Maleinsäureanhydrid (MA; protonierte Masse 99) während der Laufzeit des Experiments unter den auf der Abszisse von e) bezeichneten Bedingungen, b) Austrittsarbeit $\Phi$, c) Bindunsgenergie des V3d-Valenzzustands, d) durchschnittlicher Vanadiumoxidationszustand ( $\mathrm{V}$ ox ), berechnet aus dem V2 $\mathrm{p}_{3 / 2}$-Spektrum, und e) Elektronenaffinitätsänderung $\Delta \chi\left(\Delta \Phi-\Delta \mathrm{BE}(\mathrm{V} 3 \mathrm{~d})\right.$, Differenz zur 2. $\mathrm{O}_{2}$ Bedingung) von VPP bei $400^{\circ} \mathrm{C}$ in verschiedenen Gasmischungen. 


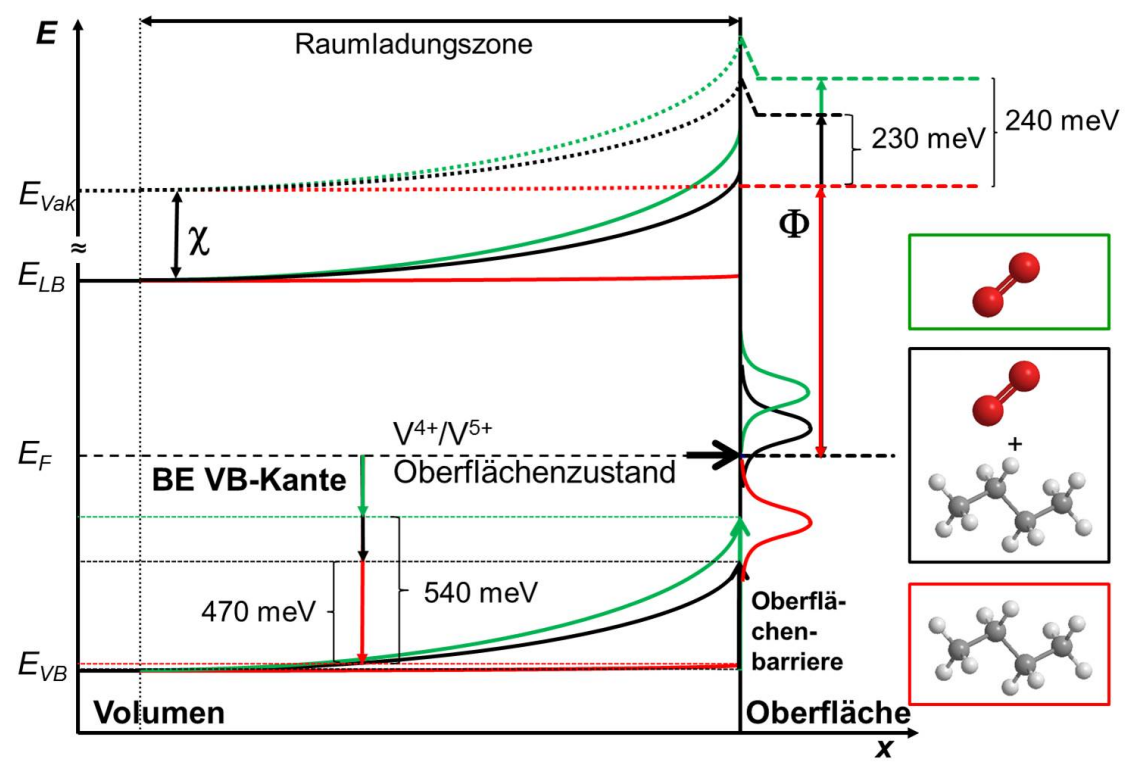

Figure 3: Schematisches Banddiagramm von VPP mit den experimentell erhaltenen Werten für die Bindungsenergie(BE)änderungen der Valenzbandkante (VB) und der Austrittsarbeit $\Phi$ gemessen in n-Butan/Helium (rot), n-Butan/Sauerstoff (schwarz), Sauerstoff/Helium (grün). $E_{V B}$ : Energie der Valenzbandkante, $E_{F}$ : Fermi-Energie, $E_{L B}$ : Energie des Leitungsbandminimums, $E_{V a k}$ : Energie des Vakuumlevels, $\chi$ : Elektronenaffinität.
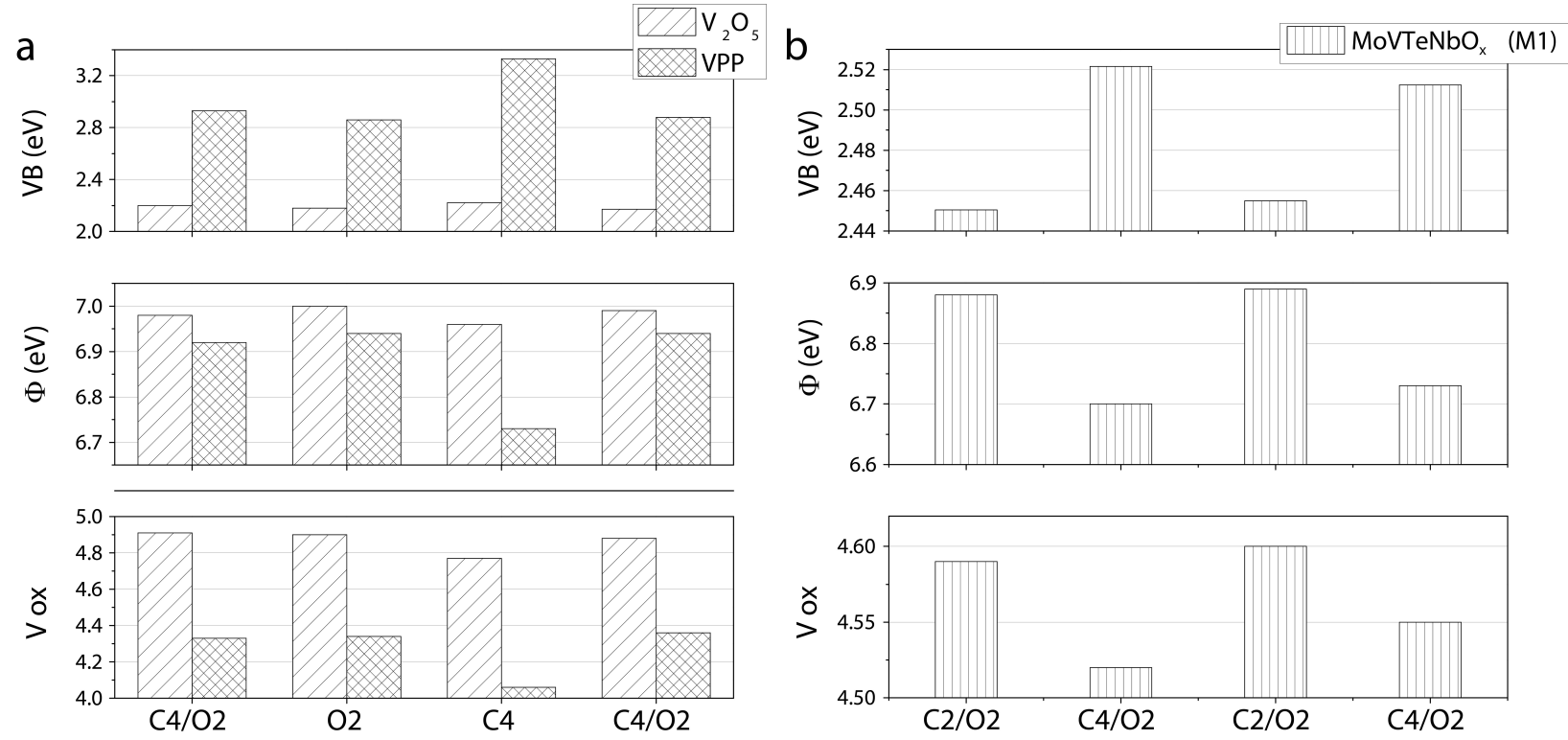

Figure 4: Valenzbandkante (VB), Austrittsarbeit $\Phi$ und Vanadiumoxidationszustand (V ox) auf der Oberfläche von VPP, $\mathrm{V}_{2} \mathrm{O}_{5}$ (a) und der $\mathrm{MoVTeNbO}_{x} \mathrm{M} 1$-Phase (b) bei $400^{\circ} \mathrm{C}$ in verschiedenen Gasmischungen. 